\title{
1 Automatic lameness detection based on 2 consecutive 3D-video recordings
}

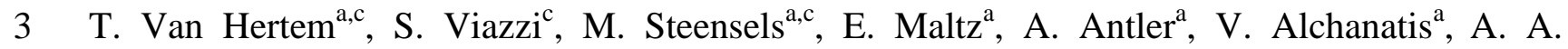
4 Schlageter-Tello ${ }^{\mathrm{b}}$, C. Lokhorst ${ }^{\mathrm{b}}$, C. E. B. Romanini ${ }^{\mathrm{c}}$, C. Bahr ${ }^{\mathrm{c}}$, D. Berckmans ${ }^{\mathrm{c}}$, and I. $5 \quad$ Halachmi $^{\mathrm{a} 1}$

$6{ }^{\mathrm{a}}$ Institute of Agricultural Engineering - Agricultural Research Organization (ARO) - the Volcani

7 Center, PO Box 6, Bet-Dagan IL-50250, Israel

$8 \quad{ }^{\mathrm{b}}$ WageningenUR Livestock Research, P.O. Box 65, NL-8200AB Lelystad, Netherlands

$9{ }^{\mathrm{c} D i v i s i o n}$ M3-BIORES: Measure, Model \& Manage Bioresponses, KU Leuven, Kasteelpark

10 Arenberg 30 - bus 2456, BE-3001 Leuven, Belgium

12 *Corresponding author:

13 Ilan Halachmi

14 Institute of Agricultural Engineering - Agricultural Research Organization (ARO) - the 15 Volcani Center,

16 P.O.Box 6, Bet Dagan IL-50250, Israel

17 Email: halachmi@ volcani.agri.gov.il

18 fax.: +97249539566

19 tel.: +972506220112

\footnotetext{
${ }^{1}$ Corresponding author: halachmi@volcani.agri.gov.il
} 


\section{ABSTRACT}

22 Manual locomotion scoring for lameness detection is a time-consuming and subjective

23 procedure. Therefore, the objective of this study is to optimise the classification output of a

24 computer vision based algorithm for automated lameness scoring. Cow gait recordings were

25 made during four consecutive night-time milking sessions on an Israeli dairy farm, using a 3D-

26 camera. A live on-the-spot assessed 5-point locomotion score was the reference for the automatic

27 lameness score evaluation. A dataset of 186 cows with four automatic lameness scores and four

28 live locomotion score repetitions was used for testing three different classification methods.

29 The analysis of the automatic scores as independent observations led to a correct classification

30 rate of $53.0 \%$ on a 5-point level scale. A multinomial logistic regression model based on four

31 individual consecutive measures obtained a correct classification rate of $60.2 \%$. When allowing a

321 unit error on the 5-point level scale, a correct classification rate of $90.9 \%$ was obtained. Strict

33 binary classification to Lame vs. Not-Lame categories reached $81.2 \%$ correct classification rate.

34 The use of cow individual consecutive measurements improved the correct classification rate of 35 an automatic lameness detection system.

36 Key words. dairy cow, lameness, computer vision, classification, consecutive measurements 


\section{1. Introduction}

38 Lameness is a major welfare issue in modern intensive dairy farming (Lievaart and Noordhuizen,

39 2011; Bruijnis, Beerda, Hogeveen \& Stassen, 2012). Liveweight (Alawneh, Stevenson,

40 Williamson, Lopez-Villalobos et al., 2012), milk fat (van Straten, Siani \& Bar, 2011), milk yield

41 (Green, Hedges, Schukken, Blowey et al., 2002; Archer, Green \& Huxley, 2010; Reader, Green,

42 Kaler, Mason et al., 2011) and behavioural indicators such as activity and lying time (Blackie,

43 Amory, Bleach \& Scaife, 2011; Pavlenko, Bergsten, Ekesbo, Kaart et al., 2011; Reader et al.,

44 2011) were all affected when a lameness incident occurred. Prevalence rates are influenced by

45 housing (Potterton, Green, Harris, Millar et al., 2011), farm management practices (Chapinal,

46 Barrientos, von Keyserlingk, Galo et al., 2013), feed (Amory, Kloosterman, Barker, Wright et

47 al., 2006) and breed (Barker, Leach, Whay, Bell et al., 2010). Averaged reported lameness

48 prevalence rates range from $20 \%$ to $25 \%$ in USA, and $33 \%$ to $37 \%$ in Europe (Schlageter Tello,

49 Bokkers, Groot Koerkamp, Van Hertem et al., 2013a). Herd locomotion scoring is a common

50 method to obtain a lameness prevalence rate (Flower \& Weary, 2009). However, this procedure

51 is time-consuming and subjective. It is therefore hardly done in practice, and when done for large

52 herd sizes, it is often done on a subsample of the entire herd (Thomsen, 2009; Main, Barker,

53 Leach, Bell et al., 2010).

54 In the scientific community, different approaches have been developed to automate locomotion 55 scoring and lameness detection. Studies using force plates (Pastell, Hautala, Poikalainen, Praks et 56 al., 2008; Liu, Dyer, Neerchal, Tasch et al., 2011; Ghotoorlar, Ghamsari, Nowrouzian \&

57 Ghidary, 2012), pressure sensitive walkways (Maertens, Vangeyte, Baert, Jantuan et al., 2011)

58 and accelerometers (Pastell, Tiusanen, Hakojarvi \& Hanninen, 2009; Chapinal, De Passille, 
59 Pastell, Hanninen et al., 2011) have measured the asymmetry of the gait when walking. These

60 approaches used the diversity in weight bearing on each leg for lameness detection.

61 The use of (mostly behavioural) sensors is spreading in modern dairy farming and there are

62 studies that use existing sensor data on the farm such as lying time (Ito, von Keyserlingk,

63 LeBlanc \& Weary, 2010), feeding behaviour and neck activity (Kramer, Cavero, Stamer \&

64 Krieter, 2009), and ruminating time, neck activity and milk yield (Van Hertem, Maltz, Antler,

65 Schlageter Tello et al., 2013) for lameness detection.

66 Other studies have used two-dimensional (2D) computer vision to analyse gait. These studies

67 focused on the measurement of different gait and posture variables such as back arch curvature

68 (Poursaberi, Bahr, Pluk, Van Nuffel et al., 2010; Viazzi, Bahr, Schlageter Tello, Van Hertem et

69 al., 2014), step overlap (Pluk, Bahr, Leroy, Poursaberi et al., 2010), hoof release angles (Pluk,

70 Bahr, Poursaberi, Maertens et al., 2012), the body movement pattern (Poursaberi, Bahr, Pluk,

71 Veermae et al., 2011). The challenge to practical application of this method is to identify

72 accurately the location in the image of anatomical body parts such as hooves, limb joints, withers

73 and back contour lines. Until now, this has been performed using manually labelled markers

74 attached to the limbs of the cows (Aoki, Kamo, Kawamoto, Zhang et al., 2006; Song, Leroy,

75 Vranken, Maertens et al., 2008; Blackie, Bleach, Amory \& Scaife, 2011). The manual labelling

76 step inhibits full automation. Video pre-processing provides an alternative for locating the

77 anatomical body parts in the video. During pre-processing, videos are transformed to sequences

78 of binary images in which anatomical parts of cows can be clearly segmented from the

79 background. Van Hertem, Alchanatis, Antler, Maltz et al. (2013) showed however that image

80 segmentation in 2D RGB-images was problematic in real farm conditions due to dynamic

81 background restrictions in side view perspective. To overcome these restrictions, a three- 
82 dimensional (3D) camera in top view perspective was suggested to obtain the same body

83 movement pattern as with the side view camera. Viazzi, Bahr, Van Hertem, Schlageter Tello et

84 al. (2014) developed an automated lameness scoring algorithm based on 3D imaging of the

85 cow's gait. The algorithm calculated the back posture measurement, which was related to the

86 back arch curvature, one of the key indicators of cow lameness (Sprecher, Hostetler \& Kaneene,

87 1997). The algorithm was validated on a small dataset of 92 cows and obtained an accuracy of

$8890 \%$ (Viazzi, Bahr, Van Hertem, et al., 2014).

89 In order to cope with false alarms - for instance when a cow trips or slips - an optimisation of the

90 algorithm is necessary. A decision made over several consecutive measurements should reduce

91 false alarms and optimise classification performance. Therefore, the aim of this study was to

92 improve the algorithm developed by Viazzi, Bahr, Van Hertem, et al., (2014) and to optimise the

93 classification performance by taking into account consecutive measurements.

\section{2. Materials and methods}

\section{$95 \quad$ 2.1.Nomenclature}

96 - BPM: Back Posture Measurement;

97 - LS: Locomotion Score;

$98 \quad$ - AS: $\quad$ Algorithm Score;

99 - 5-point classification: the classification when model and reference are subdivided

$100 \quad$ in five classes;

101 - Binary classification: the classification when model and reference are transformed from the 5-point classification to a Lame vs. Not Lame scale;

103 - 'Strict': the exact agreement between model and reference; 
the acceptable one single unit difference between model and reference;

- CCR:

107

124 avoid group mixing. All cows were fed a total mixed ration according to NRC recommendations 125 (NRC, 2001). The last six groups of the milking herd (approximately 500 cows) were followed. 126 These groups consisted of multiparous cows and lameness prevalence was highest in these correct classification rate or model accuracy;

mean absolute error;

root mean squared deviation;

contingency coefficient;

the ability to correctly classify Lame cows;

the ability to correctly classify Not Lame cows;

the tolerant misclassification rate or the misclassifications with a difference between model and reference bigger than one unit.

\subsection{Animals and housing}

Data were obtained by the Agricultural Research Organization (ARO) in a commercial dairy farm in kibbutz Yifat, Israel. The milking herd consisted of 1100 Israeli-Holstein dairy cows. The herd contained 12 production groups according to parity, lactation stage, reproduction status and health status (group size: $96 \pm 12$ cows). All cows were housed in separate no-stall fully roofed open cowsheds with dried manure bedding material. Lameness prevalence ranged between $15 \%$ and $25 \%$ throughout the year.

All cows were milked three times a day (start at $0300 \mathrm{~h}, 1100 \mathrm{~h}$ and $1900 \mathrm{~h}$ ) in a 2x32 side-byside milking parlour. The production groups were brought one-by-one to the waiting parlour to 
127 groups. The same procedure was done by Viazzi, Bahr, Van Hertem et al. (2014) on the same 128 farm.

\subsection{D camera setup}

130 An after-milking sorting gate was the only place on the farm that all cows passed. The 3D131 camera was located after the sorting gate. A wide lane allowed heavy cow traffic to the milking 132 parlour. In order to make the cows walk in the camera field of view, a mobile narrow corridor

133 (maximum width $=2.10 \mathrm{~m}$; minimum width $=1.00 \mathrm{~m}$ ) was built directly after the sorting gate

134 (Figure 1). A static corridor would interfere with the bi-weekly manure scraping in the lanes. The 135 sorting gate and the $90^{\circ}$ turn in the corridor provided the necessary time delay between 136 successive cows. In the corridor, the cows walked in a straight line and behind each other. The 137 corridor was built to obtain smooth cow traffic and to avoid queuing as much as possible in real 138 farm conditions.

140 (Insert Figure 1 here)

142 Cow gait was recorded with a Microsoft Kinect Xbox 3D-camera (Kinect ${ }^{\mathrm{TM}}$, Microsoft corp.,

143 Washington, USA). The camera was positioned in top down perspective, $3.20 \mathrm{~m}$ above ground 144 level (Figure 1), in order to have the full cow body (head-tail length range: $2.44 \mathrm{~m}-2.80 \mathrm{~m}$ ) 145 visible in the camera field of view at 30 fps. A photocell (HRTL 96B ${ }^{\mathrm{TM}}$, Leuze electronic $146 \mathrm{GmBH}$, Owen, Germany) was used to trigger the video recording. This photocell was located 0.5

$147 \mathrm{~m}$ before the beginning of the camera field of view, and was linked to a programmable logic 148 controller (NI USB-6501, National Instruments, Austin Texas, USA). The controller was set to 149 record four seconds in order to have only one cow per video. The camera was connected to an 
150 operating computer through a USB-port. After each data collection session, the construction was

151 packed away along the walking lane path, where it did not interfere with the farm routine. The

152 recorded videos contained a depth recording (for 3D-reconstruction) and a RGB-recording and

153 were saved as .oni-files to a 1TB hard disk (Western Digital, Irvine California, USA). The

154 OpenNI 1.0 Software Development Kit framework (www.openni.org, last accessed at 24 March

155 2012) was used to make recordings with the Kinect camera.

156 Due to the sunlight sensitivity of the cameras, data were collected during four consecutive night

157 milking sessions. External artificial light sources were installed around the video corridor, but

158 not pointing directly to the sensor, to increase cow visibility for locomotion scoring and visual

159 identification.

$160 \quad$ 2.4.Locomotion score reference

161 During each data collection period, the locomotion of cows passing the corridor was manually

162 scored on-the-spot by the same one trained observer (so called 'locomotion score', 'LS' or

163 'reference'). The locomotion scoring was based on the discrete 5-point numerical score of

164 Sprecher, Hostetler and Kaneene (1997) [1=healthy; 5= severely lame].

165 The intra-observer repeatability of the trained observer for the 5-point score was quantified and

166 achieved a kappa coefficient (based on Cohen (1960)) of $0.53 \pm 0.02$, and a weighed kappa

167 coefficient of $0.69 \pm 0.03$ in the four consecutive locomotion scoring sessions. These intra-

168 observer repeatability values indicate substantial repeatability (Landis \& Koch, 1977), and proof

169 of the level of training.

$170 \quad$ 2.5. 3D video analysis

171 All recorded videos were analysed automatically with a software program. The software 172 included the algorithm for the extraction of the four image features described by Viazzi, Bahr, 
173 Van Hertem et al. (2014) from the depth images. On top of that, the four parameters were

174 combined in one output variable "Back Posture Measurement" (BPM) by a weighing function

175 similar to the function described in Viazzi, Bahr, Van Hertem et al. (2014) for 2D-images. The

176 software was compiled with a MATLAB Runtime Compiler (Matlab® R2011b, The

177 MathWorks $\odot$, Inc, Natick, MA, United States) and executed on the recording computer after

178 video recording. The input of this software was the Kinect output video in .oni-format. The

179 software analysed all depth-frames in which a full cow body shape could be segmented. On

180 average $4.9 \pm 2.7$ frames per video were analysed, depending on the walking speed of the cow.

181 The output was a MATLAB-file including all usable depth-frames for analysis, together with the

182 associated RGB-frames, and the generated BPM-value related to the cow's back posture in the

183 video. The BPM-output was calculated as the median value of all frames in the video that were

184 stored in the MATLAB-file. The BPM-output in this herd ranged from 0.13 to 0.33 ..

185 The cows in the video were manually identified based on the recorded RGB-video frames. The

186 video timestamp was used to double check cow identification with the automatically generated

187 cow list in the sorting gate when the numbers on the back were hard to read.

188 At the end of the analysis, a report was generated containing cow number, the number of usable 189 frames in the video related to the cow number, and the BPM-value.

\section{$190 \quad$ 2.6.Data selection}

191 In four consecutive night milking sessions, 1327 complete cow-observations were done on 511

192 individual cows. A complete observation consisted of a live locomotion score by the observer

193 and a successfully recorded video. A subset of 186 individual cows that were identified in all

194 four consecutive sessions ( $4 \times 186=744$ cow-observations) was selected for further analysis. The

195 three selection criteria were that (i) four consecutive locomotion scores were available; (ii) four 
196 consecutive videos were available; (iii) the four consecutive locomotion scores did not vary more

197 than one numerical unit to reduce human errors in the reference. The first two criteria reduced

198 the dataset to 195 individual cows and 780 cow-observations. The last criteria was not met by 9

199 individual cows, and therefore 36 more cow observations were omitted. This implied that 583

200 cow-observations (44\%) were omitted for further analysis.

\subsection{Classification procedures}

\subsubsection{Independent cow observations.}

203 The AS was compared to LS. In order to put the BPM-score on the same scale as the LS [classes 204 1-5], a rescaling of the BPM-values was done with Equation 1, with $\min (B P M)$ the minimum 205 value of BPM, and $\max (B P M)$ the maximum value of BPM.

$$
A S=0.5+5^{*}((B P M-\min (B P M)) /(\max (B P M)-\min (B P M)))
$$

207 The AS-values were transformed to their nearest integer values.

208 Another approach to put the BPM-score on the same scale as the LS was using four non209 equidistant cut-off thresholds. For each combination of cut-off thresholds in the range of $210[\min (B P M), \max (B P M)]$, the $\mathrm{CCR}$ and MCR were calculated. The four thresholds that 211 maximised the 5-point CCR were selected as the maximising CCR-thresholds. The thresholds 212 that minimised the 5-point MCR were selected as the minimising MCR-thresholds.

\subsubsection{Models with individual consecutive measurements.}

214 Instead of analysing all measurements as independent observations, the 744 cow-observations

215 were considered as four consecutive measures of the same 186 individual cows. Three different 216 classification models were developed and compared to each other, by using the MATLAB 
217 R2011b Statistics Toolbox. These models take into account multiple consecutive readings before 218 making a final classification.

219 Classification models. For model calibration, 2/3 of the data were used, and the remaining $1 / 3$ of 220 the data were used for model validation. The model calibration and validation dataset had an 221 equal proportion and distribution according to the reference data. The rounded average rounds

222 the decimal number to the nearest integer value. If the decimal of the average value of four 223 consecutive measurements was equal to 0.5 , the rounded average was rounded downward if the 224 last measurement was equal to the lowest value in the range, and upwards if equal to the highest 225 value in the range.

226 - An ordinal multinomial logistic regression model was used because the rounded average

227 of four consecutive locomotion scores, used as reference, was interpreted as an ordinal 228 outcome variable according to lameness severity. The model allows multiple discrete 229 outcomes, in order to predict the probabilities of the different possible outcomes of a 230 categorical outcome variable, given a set of four consecutive BPM measurements as 231 input variables (Hosmer \& Lemeshow, 2000).

232 - When a nominal multinomial logistic regression model was used, the rounded average of 233 four consecutive locomotion scores, used as reference, was interpreted as a nominal 234 outcome variable with no relationship between the different categorical classes. The 235 model allows multiple discrete outcomes, in order to predict the probabilities of the 236 different possible outcomes of a categorical outcome variable, given a set of four 237 consecutive BPM-scores as input variables (Hosmer \& Lemeshow, 2000).

238 - A linear regression model was used when the reference, the average of four consecutive 239 locomotion scores, was considered as a continuous variable. The model makes a 
weighted sum of the four consecutive BPM input values, in such a way that it would fit the output variable. The weights are determined by applying the least squares method to the calibration dataset (Neter, Kutner, Nachtsheim \& Wasserman, 1996).

243 Improving model robustness. In order to obtain more robust model outcomes, a cross-validation 244 and a bootstrap aggregating procedure were used.

245 - All models were validated using a 5-fold $(k=5)$ cross-validation procedure on the 186

\subsubsection{Confusion matrix}

261 A confusion matrix was used to evaluate the classification model output against the LS 262 reference. 
263 5-point classification. In the 5-point confusion matrix, both the reference and the model output 264 are tabulated in 5 levels. The CCR is defined as the sum of the elements on the main diagonal in

265 the confusion matrix. Besides CCR, the 5-point classification performance is also expressed by

266 the mean absolute error (MAE), the root mean squared deviation (RMSD) and the contingency

267 coefficient (CC) of the confusion matrix.

268 Strict binary classification. The 5-point locomotion score was transformed to a binary score 269 (Lame vs. Not-Lame). Cows that were scored as $\mathrm{LS}=1$ or $\mathrm{LS}=2$, were considered to be Not270 Lame, and cows that were scored as $\mathrm{LS}=3, \mathrm{LS}=4$ or $\mathrm{LS}=5$ were considered to be Lame.

\section{3. Results}

\subsection{Classification of independent cow-observations}

- Applying thresholds that maximise 5-point $\mathrm{CCR}(\mathrm{T} 1=0.16, \mathrm{~T} 2=0.21, \mathrm{~T} 3=0.25$ and $\mathrm{T} 4=0.30)$ resulted in a correct classification rate of $53.0 \%$ (Table 1a).

- Applying thresholds that minimise 5-point $\mathrm{MCR}(\mathrm{T} 1=0.14, \mathrm{~T} 2=0.24, \mathrm{~T} 3=0.26$ and $\mathrm{T} 4=0.30$ ) resulted in a correct classification rate of $42.6 \%$ (Table $1 \mathrm{~b}$ ).

- Applying equidistant thresholds after rescaling $(\mathrm{T} 1=0.17, \mathrm{~T} 2=0.21, \mathrm{~T} 3=0.25$ and $\mathrm{T} 4=0.29)$ resulted in a correct classification rate of $52.6 \%$ (Table $1 \mathrm{c}$ ). 


\subsection{Bootstrap voting classification of the nominal multinomial logistic} regression model with individual consecutive measurements

283 Strict classification. The 5-point confusion matrix is presented in Table 2. Bootstrap voting on

284 the 5-fold cross validated ordinal multinomial logistic regression model resulted in a CCR of $285 \quad 60.2 \%$ (Table 4a).

287 (Insert Table 2 here)

289 Strict binary classification results are presented in Table 3, and reached a CCR of $81.2 \%$ (Table 290 4f). Sensitivity of strict binary classification was $47.1 \%$, and specificity was $94.1 \%$ (Table 4f).

292 (Insert Table 3 here)

293

294 Tolerant classification. When allowing a 1 unit error, the 5-point CCR was 90.9\% (Table 4b).

295 The MAE is 0.500 , the RMSD is equal to 0.852 and the $\mathrm{CC}$ is equal to 0.791 . measurements

5-point classification. The tolerant CCR was comparable for all three models $(90.9 \%, 91.4 \%$ and 91.9\%; Table 4b). The linear regression model had the lowest strict CCR (56.5\%; Table 4a)

302 Binary classification. Regarding the strict binary classification of lameness, the ordinal 303 multinomial logistic regression model had a higher CCR (81.2\%; Table 4f) than the nominal 
304 multinomial logistic regression model (80.7\%) and the linear regression model (80.7\%). The best

305 sensitivity value was obtained with the linear regression model (54.9\%), which was higher than

306 the sensitivity of the nominal and ordinal multinomial logistic regression models (47.1\%). Model

307 specificity was higher for the ordinal multinomial logistic regression model (94.1\%) than for the

308 nominal multinomial logistic regression model (93.3\%) and the linear regression model (90.4\%).

310 (Insert Table 4 here)

\section{4. Discussion}

312 The results suggest that an accurate lameness detection can be made by applying a 3D-camera

313 and a multinomial logistic regression of four consecutive measurements. Independent analysis of

314 the 3D-algorithm output was not good enough $(\mathrm{CCR}=53.0 \%$ on a 5-point level).

315 Taking into account four measurements by applying a classification model such as the ordinal 316 multinomial logistic regression, the correct classification rate was improved to $60.2 \%$. Three 317 different classification models were tested in this study, and they differed slightly in handling the 318 reference. The four consecutive measurements were considered as dependent repetitions of the 319 same individual cow, while in the first approach, all measurements were analysed independently. 320 Consecutive measurement analysis requires an established database while independent analysis 321 can deliver answers immediately on-the-spot without the need to establish a database.

322 Making use of consecutive measurements increased the certainty of the model in order to avoid 323 presenting false alarms to the farmers. De Mol, Bleumer, van der Werf and Van Reenen (2012) 324 used a similar approach based on seven consecutive days. In this study, neither the optimal 325 classification model settings nor the optimal number of consecutive measurements were tested 326 for obtaining the best classification results. In further research, the independent analysis should 
327 be further developed, perhaps after improving the BPM value by applying other filters and image

328 processing techniques.

329 Improvement of CCR from $60.2 \%$ to $81.2 \%$ was achieved when transforming the 5-point scale to

330 a strict binary scale (Lame vs. Not-Lame), a method previously applied by Winckler and Willen

331 (2001), Channon, Walker, Pfau, Sheldon et al. (2009) and Main et al. (2010). A binary score is

332 simple and easy to understand and it gives an agronomic value to the algorithm output. For

333 practical use, the farmer needs to be informed about which cows are lame and need treatment,

334 and which are not lame. On the other hand, a binary classification hides some useful information.

335 A commonly used cut-off threshold to differentiate between clinical and subclinical lame cows is

336 between 2 and 3 (Winckler \& Willen, 2001). Cows that were scored as 2 or 3 by the reference or

337 the model had a larger impact on the strict classification, whereas for the tolerant classification

338 these values were still acceptable.

339 In the presented analysis, cut-off thresholds were determined at a group level. In further research

340 when more consecutive measurements can be available, cow-specific individual cut-off

341 thresholds should be calculated. Viazzi, Bahr, Schlageter-Tello et al. (2013) have shown that

342 using an individual threshold on a BPM time series can improve the accuracy of the model by

$34310 \%$.

344 In this study, a 5-point live locomotion scoring (Sprecher, Hostetler \& Kaneene, 1997) was

345 performed on-the-spot, and served as the reference. This 'gold' standard is known to be

346 subjective and inter- and intra-observer repeatability is low (Flower \& Weary, 2009; Schlageter

347 Tello, Bokkers, Groot Koerkamp, Van Hertem et al., 2013b). Locomotion scoring is however a

348 commonly used method because it provides an immediate, on-site assessment and it does not

349 require technical equipment (Flower \& Weary, 2009). As a first tentative approach to achieve 
350 higher reliability in the reference and reduce the subjectivity effect of the scorer, only cows that 351 had four consecutive live scorings within a one unit score-range were selected ( $\mathrm{n}=186$ cows)

352 before the analysis. A second tentative suggestion was allowing a 1 unit error in the 5-point 353 scale, and this led to a tolerant CCR of $90.9 \%(n=169$ cows). A MAE of 0.50 shows that the 5354 point classification is acceptable.

355 The accuracy of the strict binary classification, $\mathrm{CCR}=81.2 \%$, is lower than the accuracy 356 obtained by Viazzi, Bahr, Van Hertem, et al. (2014), who achieved a CCR of 90\%. Viazzi, Bahr, 357 Van Hertem, et al. (2014) gathered their data in one single evening milking session, whereas the 358 data in this analysis were gathered on four consecutive evening milking sessions. This may 359 suggest that the daily reinstallation of the camera might influence the recording.

360 The comparison between the three classification models revealed that the linear regression model 361 (54.9\% vs. $47.1 \%$ for the multinomial logistic regression models) obtained higher sensitivity 362 values on a binary scale than the multinomial logistic regression models. For the 5-point scale 363 however, the multinomial logistic regression model (60.2\%) performed better than the linear 364 regression model (56.5\%). Depending on the desired outcome (5-point scale or binary scale), one 365 can choose the best classification model.

366 It is important to compare the results in our study with other studies. Care should be taken when 367 comparing tolerant results to strict outcomes since they are not the same. Tolerant analysis 368 allows a one unit error in the outcome, whereas the strict analysis only allows the exact 369 agreement between the outcome and the reference. The CCR in our study (CCR $=90.9 \%)$ was 370 higher in comparison to the GAITWISE system (Maertens et al., 2011) $(\mathrm{CCR}=133 / 159=$ $37183.7 \%$ ) which was based on kinematic variables. GAITWISE reached a model sensitivity of 76$37290 \%$, and a model specificity of $86-100 \%$ for a three level gait score. In the setup, they needed a 
373 large separation between the animals in order not to have two cows on the pressure sensitive

374 walkway at the same time. In our study, a separation between the animals was also necessary,

375 but a small separation was sufficient to automatically differentiate consecutive cows during

376 image segmentation. GAITWISE's lowest sensitivity values were obtained for the middle class

377 scored cows (76\%), indicating the biggest difficulty is detecting the mildly lame animals. These

378 results compare to the results in this study, where the cows scored as LS $=2$ and LS $=3$ have a

379 large impact on binary model classification accuracy.

380 Poursaberi et al. (2010) obtained an accuracy of $96 \%$ on a dataset of 184 cows. Their algorithm

381 analysed the back curvature with the inverse radius variable on 2D side view images. Their side

382 view video recordings however were made in controlled experimental conditions on an

383 experimental dairy farm - not on a commercial dairy farm.

384 In this semi-automatic setup, the video recording and analysis were done fully automatically.

385 The identification of the cows in the videos was however done manually, after the videos were

386 recorded. A time delay between the cows is only necessary for the image processing in the

387 analysis phase. The time to analyse each individual video is rather small ( $<10$ seconds). In a

388 fully automated setup (recording + identification + analysis) that is also integrated into the farm

389 management software, it would be possible to operate a separation fence to sort the lame cows

390 from the non-lame cows. It is however advised to have some distance (at this moment the ideal

391 distance is unknown) between the recording spot and the separation fence. This allows more time

392 for recorded video analysis, transforming the system outcome to a sorting gate input signal and

393 hence smoother cow traffic. The real implementation in farm conditions and what is the best

394 way to use the system should be further investigated. 
395 The recordings in this study were made during night time milking sessions due to the sunlight 396 sensitivity of the camera. The corridor was built in an unroofed part of the farm. If however night

397 milking sessions are not available, recordings could also be made in shaded areas where the

398 direct or diffuse sunlight is low, because it is the infrared spectrum of the sunlight that affects

399 camera performance. The system is therefore also applicable in other dairy husbandry systems.

400 The automatic scoring in our study only focused on the arching of the cow's back. Detecting 401 only the back arching as a method to detect lameness has been described earlier by Poursaberi et 402 al. (2010), Poursaberi et al. (2011) and Viazzi, Bahr, Schlageter Tello, et al. (2014). However, 403 when performing a locomotion scoring, the back arching is only one of the indicators (Flower \& 404 Weary, 2009; Schlageter Tello, Lokhorst, Van Hertem, Halachmi et al., 2011). In further 405 research, it is advised that other parameters such as gait asymmetry and head bob should also be 406 included in the 3D-video analysis.

407 Van Hertem et al. (2013b) developed an automatic lameness detection model based on 408 behavioural and performance variables that reached $85 \%$ model sensitivity and $89 \%$ model 409 specificity. Future research is needed to reveal whether the combination of both approaches 410 (computer vision, and behaviour and performance sensing) increases the classification accuracy 411 of the combined model, and which variables should be included in the combined model.

\section{5. Conclusions}

413 A 3D-video based algorithm for lameness detection was validated in real farm conditions and 414 compared with live locomotion scoring.

415 - Independent cow-observation analysis resulted in a correct classification rate of $53.0 \%$.

416 - When four individual consecutive measurements were taken into account in a 417 multinomial logistic regression model, a correct classification rate of $60.2 \%$ was reached. 
- After transforming the algorithm output to a strict binary scale in order to give it a biological meaning, a correct classification rate of $81.2 \%$ was obtained.

- When allowing a one unit error as a tentative approach to reduce observer-related errors, a tolerant correct classification rate of $90.9 \%$ was obtained.

423 classification output of a computer vision system. This conclusion should be considered before 424 implementing an automatic lameness detection system on more farms.

\section{Acknowledgements}

426 The authors thank all farm personnel for their help on the farm. The authors thank Uzi Birk and 427 Daniel Rozen from DeLaval for their help with the product development. The help from Doron 428 Bar, Rony Meir and staff from SCR is also very much appreciated. The authors thank V. 429 Ostrovsky for his help with the setup.

430 This study is part of the Marie Curie Initial Training Network BioBusiness (FP7-PEOPLE-ITN431 2008). This study is contribution number 459-4398-951, funded by the Agricultural Research 432 Organization (ARO), P.O.Box 6, Bet Dagan, Israel.

\section{References}

Alawneh, J. I., M. A. Stevenson, N. B. Williamson, N. Lopez-Villalobos \& T. Otley. 2012. The effect of clinical lameness on liveweight in a seasonally calving, pasture-fed dairy herd. Journal of Dairy Science 95(2):663-669.

Amory, J. R., P. Kloosterman, Z. E. Barker, J. L. Wright, R. W. Blowey, et al. 2006. Risk factors for reduced locomotion in dairy cattle on nineteen farms in the Netherlands. Journal of Dairy Science 89(5):1509-1515.

Aoki, Y., M. Kamo, H. Kawamoto, J. Zhang \& A. Yamada. 2006. Changes in walking parameters of milking cows after hoof trimming. Animal Science Journal 77(1):103-109.

Archer, S. C., M. J. Green \& J. N. Huxley. 2010. Association between milk yield and serial locomotion score assessments in UK dairy cows. Journal of Dairy Science 93(9):4045-4053. 
Barker, Z. E., K. A. Leach, H. R. Whay, N. J. Bell \& D. C. J. Main. 2010. Assessment of lameness prevalence and associated risk factors in dairy herds in England and Wales. Journal of Dairy Science 93(3):932-941.

Blackie, N., J. R. Amory, E. Bleach \& J. Scaife. 2011. The effect of lameness on lying behaviour of zero grazed Holstein dairy cattle. Applied Animal Behaviour Science 134(3):85-91.

Blackie, N., E. Bleach, J. R. Amory \& J. Scaife. 2011. Impact of lameness on gait characteristics and lying behaviour of zero grazed dairy cattle in early lactation. Applied Animal Behaviour Science 129(2-4):67-73.

Breiman, L. 1996. Bagging predictors. Machine learning 24(2):123-140.

Bruijnis, M. R. N., B. Beerda, H. Hogeveen \& E. N. Stassen. 2012. Assessing the welfare impact of foot disorders in dairy cattle by a modeling approach. Animal 6(6):962-970.

Channon, A. J., A. M. Walker, T. Pfau, I. M. Sheldon \& A. M. Wilson. 2009. Variability of Manson and Leaver locomotion scores assigned to dairy cows by different observers. Veterinary Record 164(13):388-392.

Chapinal, N., A. K. Barrientos, M. A. G. von Keyserlingk, E. Galo \& D. M. Weary. 2013. Herdlevel factors for lameness in freestall farms in the northeastern United States and California. Journal of Dairy Science 96(1):318-328.

Chapinal, N., A. M. De Passille, M. Pastell, L. Hanninen, L. Munksgaard, et al. 2011. Measurement of acceleration while walking as an automated method for gait assessment in dairy cattle. Journal of Dairy Science 94(6):2895-2901.

Cohen, J. 1960. A coefficient of agreement for nominal scales. Educational and Psychological Measurement 20(1):37-46.

de Mol, R. M., A. G. Bleumer, J. T. N. van der Werf \& C. G. Van Reenen. 2012. Automated detection of lameness in dairy cows based on day-to-day variation in behaviour. Page 396 in 63rd Annual Meeting of the European Federation of Animal Science (EAAP). Vol. Book of Abstracts. Wageningen Academic Publishers, Bratislava, Slovakia.

Flower, F. C. \& D. M. Weary. 2009. Gait assessment in dairy cattle. Animal 3(1):87-95.

Ghotoorlar, S. M., S. M. Ghamsari, I. Nowrouzian \& S. S. Ghidary. 2012. Lameness scoring system for dairy cows using force plates and artificial intelligence. Veterinary Record 170(5):126-153.

Green, L. E., V. J. Hedges, Y. H. Schukken, R. W. Blowey \& A. J. Packington. 2002. The impact of clinical lameness on the milk yield of dairy cows. Journal of Dairy Science 85(9):2250-2256.

Hosmer, D. W. \& S. Lemeshow. 2000. Applied Logistic Regression. John Wiley \& Sons, New York, NY, USA.

Ito, K., M. A. G. von Keyserlingk, S. J. LeBlanc \& D. M. Weary. 2010. Lying behavior as an indicator of lameness in dairy cows. Journal of Dairy Science 93(8):3553-3560.

Kramer, E., D. Cavero, E. Stamer \& J. Krieter. 2009. Mastitis and lameness detection in dairy cows by application of fuzzy logic. Livestock Science 125(1):92-96.

Landis, J. R. \& G. G. Koch. 1977. The measurement of observer agreement for categorical data. Biometrics 33(1):159-174.

Lievaart, J. J. \& J. P. T. M. Noordhuizen. 2011. Ranking experts' preferences regarding measures and methods of assessment of welfare in dairy herds using Adaptive Conjoint Analysis. Journal of Dairy Science 94(7):3420-3427. 
Liu, J. B., R. M. Dyer, N. K. Neerchal, U. Tasch \& P. G. Rajkondawar. 2011. Diversity in the magnitude of hind limb unloading occurs with similar forms of lameness in dairy cows. Journal of Dairy Research 78(2):168-177.

Maertens, W., J. Vangeyte, J. Baert, A. Jantuan, K. C. Mertens, et al. 2011. Development of a real time cow gait tracking and analysing tool to assess lameness using a pressure sensitive walkway: The GAITWISE system. Biosystems Engineering 110:29-39.

Main, D. C. J., Z. E. Barker, K. A. Leach, N. J. Bell, H. R. Whay, et al. 2010. Sampling strategies for monitoring lameness in dairy cattle. Journal of Dairy Science 93(5):1970-1978.

Neter, J., M. H. Kutner, C. J. Nachtsheim \& W. Wasserman. 1996. Applied linear regression models. Third edition ed. The McGraw-Hill Companies, Inc.

NRC. 2001. Nutrient Requirements of Dairy Cattle. Vol. 1. 7 ed. National Academies Press, Washington D.C.

Pastell, M., M. Hautala, V. Poikalainen, J. Praks, I. Veermae, et al. 2008. Automatic observation of cow leg health using load sensors. Computers and Electronics in Agriculture 62(1):48-53.

Pastell, M., J. Tiusanen, M. Hakojarvi \& L. Hanninen. 2009. A wireless accelerometer system with wavelet analysis for assessing lameness in cattle. Biosystems Engineering 104(4):545551.

Pavlenko, A., C. Bergsten, I. Ekesbo, T. Kaart, A. Aland, et al. 2011. Influence of digital dermatitis and sole ulcer on dairy cow behaviour and milk production. Animal 5(8):12591269.

Pluk, A., C. Bahr, T. Leroy, A. Poursaberi, X. Song, et al. 2010. Evaluation of step overlap as an automatic measure in dairy cow locomotion. Transactions of the Asabe 53(4):1305-1312.

Pluk, A., C. Bahr, A. Poursaberi, W. Maertens, A. van Nuffel, et al. 2012. Automatic measurement of touch and release angles of the fetlock joint for lameness detection in dairy cattle using vision techniques. Journal of Dairy Science 95(4):1738-1748.

Potterton, S. L., M. J. Green, J. Harris, K. M. Millar, H. R. Whay, et al. 2011. Risk factors associated with hair loss, ulceration, and swelling at the hock in freestall-housed UK dairy herds. Journal of Dairy Science 94(6):2952-2963.

Poursaberi, A., C. Bahr, A. Pluk, A. Van Nuffel \& D. Berckmans. 2010. Real-time automatic lameness detection based on back posture extraction in dairy cattle: Shape analysis of cow with image processing techniques. Computers and Electronics in Agriculture 74(1):110-119.

Poursaberi, A., C. Bahr, A. Pluk, I. Veermae, E. Kokin, et al. 2011. Online Lameness Detection in Dairy Cattle Using Body Movement Pattern. in 11th International Conference on Intelligent Systems Design and Applications (ISDA 2011).

Reader, J. D., M. J. Green, J. Kaler, S. A. Mason \& L. E. Green. 2011. Effect of mobility score on milk yield and activity in dairy cattle. Journal of Dairy Science 94(10):5045-5052.

Schlageter Tello, A., E. A. M. Bokkers, P. W. G. Groot Koerkamp, T. Van Hertem, S. Viazzi, et al. 2013a. Locomotion scoring as a golden standard for automated lameness detection: a review. Preventive Veterinary Medicine (submitted).

Schlageter Tello, A., E. A. M. Bokkers, P. W. G. Groot Koerkamp, T. Van Hertem, S. Viazzi, et al. 2013b. Within and between observer repeatability of live and video locomotion assessments in dairy cows. Animal Welfare (submitted).

Schlageter Tello, A., C. Lokhorst, T. Van Hertem, I. Halachmi, E. Maltz, et al. 2011. Selection of a golden standard for visual-based automatic lameness detection for dairy cows. in Animal hygiene and sustainable livestock production. Proceedings of the XVth International 
Congress of the International Society for Animal Hygiene. Vol. 1. J. Kofer \& H. Schobesberger, ed. Tribun EU, Vienna, Austria.

Song, X. Y., T. Leroy, E. Vranken, W. Maertens, B. Sonck, et al. 2008. Automatic detection of lameness in dairy cattle - Vision-based trackway analysis in cow's locomotion. Computers and Electronics in Agriculture 64(1):39-44.

Sprecher, D. J., D. E. Hostetler \& J. B. Kaneene. 1997. A lameness scoring system that uses posture and gait to predict dairy cattle reproductive performance. Theriogenology 47(6):1179-1187.

Thomsen, P. T. 2009. Rapid screening method for lameness in dairy cows. Veterinary Record 164(22):689-690.

Van Hertem, T., V. Alchanatis, A. Antler, E. Maltz, A. Schlageter Tello, et al. 2013. Comparison of segmentation algorithms for cow contour extraction from natural barn background in side view images. Computers and Electronics in Agriculture 91(2):65-74.

Van Hertem, T., E. Maltz, A. Antler, A. Schlageter Tello, C. Lokhorst, et al. 2013. Lameness detection based on multivariate continuous sensing of milk yield, rumination and neck activity. Journal of Dairy Science 96(7):4286-4298.

van Straten, M., I. Siani \& D. Bar. 2011. Reduced test-day milk fat percentage in cows diagnosed with claw horn lesions during routine claw trimming. Journal of Dairy Science 94(4):1858-1863.

Viazzi, S., C. Bahr, A. Schlageter Tello, T. Van Hertem, C. E. B. Romanini, et al. 2013. Analysis of individual classification of lameness using automatic back posture measurement in dairy cattle. Journal of Dairy Science 96(1):257-266.

Viazzi, S., C. Bahr, T. Van Hertem, A. Schlageter Tello, C. E. B. Romanini, et al. 2014. Comparison of a three-dimensional and two-dimensional camera system for automated measurement of back posture in dairy cows. Computers and Electronics in Agriculture 100(1):139-147.

Winckler, C. \& S. Willen. 2001. The reliability and repeatability of a lameness scoring system for use as an indicator of welfare in dairy cattle. Acta Agriculturae Scandinavica Section A. Animal Science(Supplementum 30):103-107. 


\section{List of Tables}

Table 1: Classification of $n=744$ independent cow-observations represented with confusion matrices. The strict correct classification rate for each confusion matrix is presented below each table.

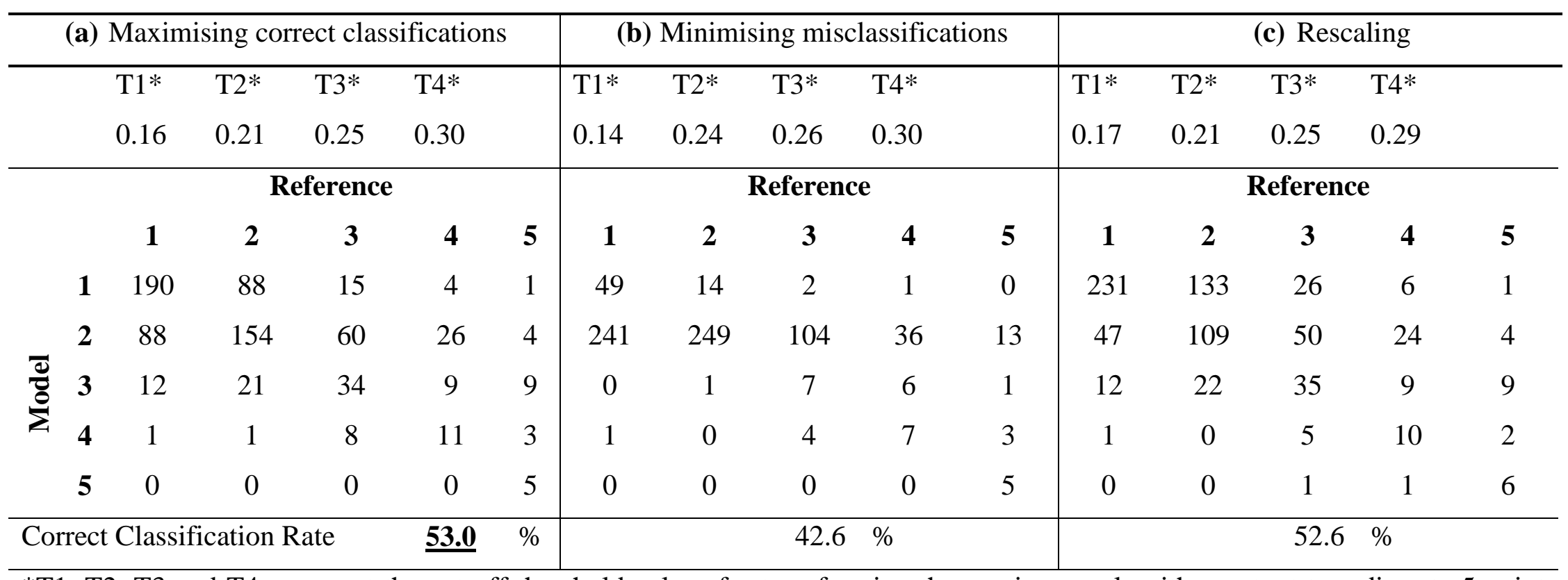

*T1, T2, T3 and T4 represent the cut-off threshold values for transforming the continuous algorithm output to a discrete 5-point scale in each categorisation. 
1 Table 2: The 5-point confusion matrix of the bootstrap voted ordinal multinomial logistic

2 regression model with four consecutive measurements of $n=186$ individual cows.

\begin{tabular}{ccccccc}
\hline & \multicolumn{6}{c}{ Reference } \\
& & $\mathbf{1}$ & $\mathbf{2}$ & $\mathbf{3}$ & $\mathbf{4}$ & $\mathbf{5}$ \\
& $\mathbf{1}$ & 47 & 18 & 4 & 1 & 0 \\
& $\mathbf{2}$ & 14 & 48 & 16 & 5 & 1 \\
$\overline{\mathbb{D}}$ & $\mathbf{3}$ & 2 & 5 & 10 & 3 & 3 \\
$\sum_{\Sigma}^{\circ}$ & $\mathbf{4}$ & 0 & 1 & 1 & 4 & 0 \\
& $\mathbf{5}$ & 0 & 0 & 0 & 0 & 3
\end{tabular}

3 
4 Table 3: The strict binary confusion matrix of the bootstrap voted ordinal multinomial logistic

5 regression model with four consecutive measurements of $n=186$ individual cows.

\begin{tabular}{cccc}
\hline & & \multicolumn{2}{c}{ Reference } \\
\hline \multirow{3}{*}{ Model } & Lame & 24 & NotLame \\
& NotLame & 27 & 127 \\
& & & \\
\hline
\end{tabular}




\section{Classification output}

\section{5-point}

Binary
(a) Strict
(b) Tolerant
(c)
(d)
(e)
(f)

\begin{tabular}{ccccccccc} 
& CCR & CCR & MAE & RMSD & CC & CCR & Sensitivity & Specificity \\
Ordinal Multinomial Logistic & 60.2 & 90.9 & 0.500 & 0.852 & 0.791 & 81.2 & 47.1 & 94.1 \\
Regression & & & & & & & & \\
Nominal Multinomial Logistic & 60.2 & 91.4 & 0.511 & 0.842 & 0.800 & 80.7 & 47.1 & 93.3 \\
Regression & & & & & & & & \\
Linear Regression & 56.5 & 91.9 & 0.522 & 0.839 & 0.694 & 80.7 & 54.9 & 90.4 \\
\hline
\end{tabular}

Strict = exact agreement between reference and model;

Tolerant $=$ acceptable one single unit difference between model and reference;

Measures of performance:

MAE = mean absolute error [score unit];

RMSD = root mean squared deviation [dimensionless];

$\mathrm{CC}=$ contingency coefficient [dimensionless];

$\mathrm{CCR}=$ correct classification rate or model accuracy, the overall ability to correctly classify Lame and Not-Lame animals [\%]; Sensitivity = the ability to detect Lame animals [\%]; 
Specificity $=$ the ability to detect Not-Lame animals [\%]; 


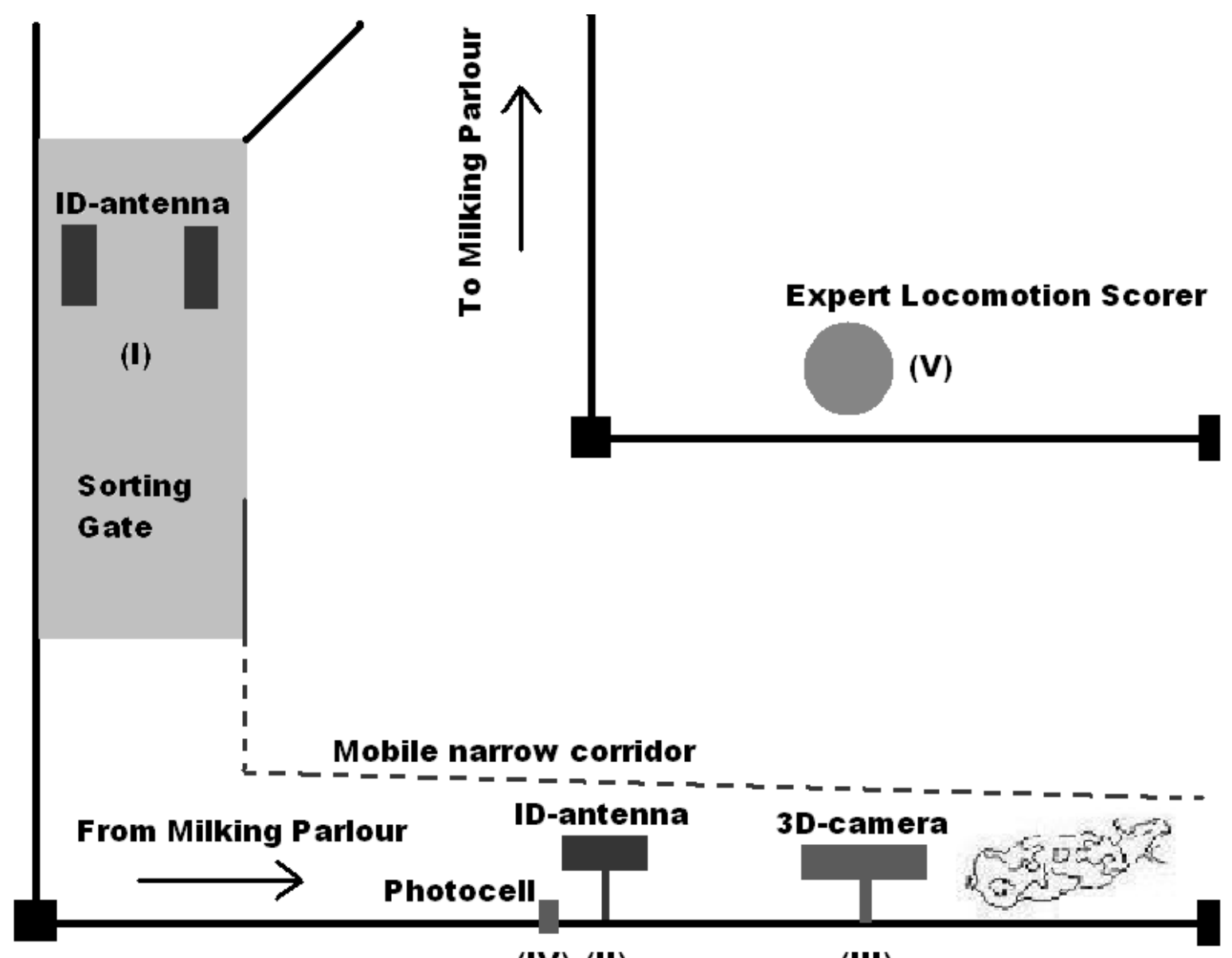

(IV) (II)

(III)

12 Figure 1: Top view layout of the 3D-camera setup. Data were collected when cows returned from

13 the milking parlour to their cowsheds. All cows were electronically identified by the antennas in

14 the sorting gate (I) and in the corridor (II). The 3D-camera (III) was triggered by the photocell

15 (IV) that was located in the corridor. A live scoring expert (V) performed a locomotion scoring

16 of the cows while they passed through the setup. 